\title{
On Bi-periodic Jacobsthal and Jacobsthal-Lucas Quaternions
}

\author{
Kubra Gul
}

Correspondence: Department of Computer Engineering, Faculty of Engineering and Architecture, Kafkas University, 36100, Kars, Turkey. E-mail: kubra.gul@kafkas.edu.tr

Received: January 7, 2019 Accepted: January 25, 2019 Online Published: February 25, 2019

doi:10.5539/jmr.v11n2p44

URL: https://doi.org/10.5539/jmr.v11n2p44

\begin{abstract}
In this paper, we introduce the bi-periodic Jacobsthal and Jacobsthal-Lucas quaternions. We give the Binet formulas and the generating functions for these quaternions. We obtain some well-known identities such as the Cassini, Catalan and D'ocagne's identities. Additionally, we give summation formulas and the relationships between bi-periodic Jacobsthal and Jacobsthal-Lucas quaternions.
\end{abstract}

Keywords: Jacobsthal sequence, Jacobsthal-Lucas sequence, generalized Jacobsthal sequence, quaternion

\section{Introduction}

Number sequences such as Fibonacci, Pell, Lucas, Jacobsthal, etc., have been studied by many authors (Horadam, 1996; Horadam, 1971; Koshy, 2001). These sequences play an important role in many fields of science (El Naschie, 2005; 2007; Kilic \& Stakhov, 2009; Stakhov, 2005). For instance, microcontrollers and other computers use conditional instructions to change the flow of execution of a program. Additionally, some microcontrollers use skip instructions that conditionally bypass the next instruction. This is useful for one case out of the four possibilities on 2 bits, 3 cases on 3 bits, 5 cases on 4 bits, 11 on 5 bits, 21 on 6 bits, 43 on 7 bits, 85 on 8 bits, etc., which are the Jacobsthal numbers (Horadam, 1996). Jacobsthal and Jacobsthal-Lucas numbers are defined by, respectively,

$J_{n}=J_{n-1}+2 J_{n-2}$, for $n \geq 2$, with initial conditions $J_{0}=0, J_{1}=1$,

$j_{n}=j_{n-1}+2 j_{n-2}$, for $n \geq 2$, with initial conditions $j_{0}=2, j_{1}=1$.

The different number sequences have many generalizations in the literature (Bilgici, 2014; Edson \& Yayenie, 2009; Horadam, 1961; Uygun, 2018; Uygun \& Owusu, 2016; 2017; Yayenie, 2011). Bi-periodic Fibonacci numbers are well-known (Edson \& Yayenie, 2009; Yayenie, 2011). Bilgici introduced bi-periodic Lucas numbers in (Bilgici, 2014). In (Uygun \& Owusu, 2016), bi-periodic Jacobsthal and in (Uygun, 2018) bi-periodic Jacobsthal-Lucas numbers were defined as, respectively,

$\hat{\jmath}_{n}=\left\{\begin{array}{ll}a \hat{\jmath}_{n-1}+2 \hat{\jmath}_{n-2}, & \text { if } n \text { is even } \\ b \hat{\jmath}_{n-1}+2 \hat{\jmath}_{n-2}, & \text { if } n \text { is odd }\end{array}\right.$ and $C_{n}=\left\{\begin{array}{ll}b C_{n-1}+2 C_{n-2}, & \text { if } n \text { is even } \\ a C_{n-1}+2 C_{n-2}, & \text { if } n \text { is odd }\end{array} \quad n \geq 2\right.$,

where $a, b$ non-zero numbers have initial values $\hat{\jmath}_{0}=0, \hat{\jmath}_{1}=1 C_{0}=2, C_{1}=a$.

In (Uygun \& Owusu, 2017), some relationships between these numbers were obtained as $C_{n}=2 \hat{\jmath}_{n-1}+\hat{\jmath}_{n+1}$ and $(a b+8) \hat{\jmath}_{n}=2 C_{n-1}+C_{n+1}$. The generating function of the sequence $\left\{\hat{\jmath}_{n}\right\}$ is $J(x)=\frac{x\left(1+a x-2 x^{2}\right)}{1-(a b+4) x^{2}+4 x^{4}}$ and the Binet formula of the sequence $\left\{\hat{\jmath}_{n}\right\}$ is $\hat{\jmath}_{n}=\left(\frac{a^{1-\epsilon(n)}}{\left.(a b)^{\frac{n}{2}}\right\rfloor}\right) \frac{\alpha^{n}-\beta^{n}}{\alpha-\beta}$, where $\epsilon(n)=n-2\left\lfloor\frac{n}{2}\right\rfloor$ expressed as $\epsilon(n)=0$ when $n$ is even and $\epsilon(n)=1$ when $n$ is odd and the roots of the poliynomial $x^{2}-a b x-2 a b$ are $\alpha$ and $\beta$ defined by

$\alpha=\frac{a b+\sqrt{a^{2} b^{2}+8 a b}}{2}$ and $\beta=\frac{a b-\sqrt{a^{2} b^{2}+8 a b}}{2}$ with the following properties:

$\alpha+\beta=a b, \alpha-\beta=\sqrt{a^{2} b^{2}+8 a b}, \alpha \beta=-2 a b$.

For further information about these sequences, see (Uygun, 2018; Uygun \& Owusu, 2016).

Quaternions were defined by Hamilton (1866) as a generalization of complex numbers. Hamilton introduced a 
quaternion in the form

$$
q=q_{0} e_{0}+q_{1} e_{1}+q_{2} e_{2}+q_{3} e_{3}
$$

where $q_{0}, q_{1}, q_{2}, q_{3}$ are real numbers. The set of quaternions is denoted by

$$
H=\left\{q=q_{0} e_{0}+q_{1} e_{1}+q_{2} e_{2}+q_{3} e_{3}: q_{s} \in \mathbb{R}, s=0,1,2,3\right\}
$$

is a 4 -dimensional $\mathbb{R}$-vector space with basis $\left\{e_{0}, e_{1}, e_{2}, e_{3}\right\}$. The basis satisfies the following:

$e_{1}^{2}=e_{2}^{2}=e_{3}^{2}=e_{1} e_{2} e_{3}=-1, e_{1} e_{2}=e_{3}=-e_{2} e_{1}, e_{2} e_{3}=e_{1}=-e_{3} e_{2}$ and $e_{3} e_{1}=e_{2}=-e_{1} e_{3}$.

$q^{*}$ the conjugate of the quaternion $q$ is defined by $q^{*}=q_{0} e_{0}-q_{1} e_{1}-q_{2} e_{2}-q_{3} e_{3}$.

The number sequences have many applications in quaternion theory. The study of the quaternions of the sequences began with the earlier work of Horadam (Horadam, 1993) where the Fibonacci quaternion was investigated. Several authors worked on different quaternions and their generalizations (Gül, 2018; Halıc1, 2012; Szynal-Liana \& Wloch, 2016; Tan, Y1lmaz \& Sahin, 2016a; Tan, Yılmaz \& Sahin, 2016b; Tasc1, 2017).

In 2016, Tan et al. defined bi-periodic Fibonacci and Lucas quaternions and obtained identities for them (Tan, Y1lmaz \& Sahin, 2016a; Tan, Yılmaz \& Sahin, 2016b). In this paper, we will introduce bi-periodic Jacobsthal and Jacobsthal-Lucas quaternions and give some of their properties.

\section{The Bi-periodic Jacobsthal and the Bi-periodic Jacobsthal-Lucas Quaternions}

Definition 2.1. The bi-periodic Jacobsthal and the bi-periodic Jacobsthal-Lucas quaternions are defined by, respectively,

$$
\hat{J}_{n}=\sum_{i=0}^{3} \hat{J}_{n+i} e_{i} \text { and } \hat{C}_{n}=\sum_{i=0}^{3} C_{n+i} e_{i}
$$

where $\hat{\jmath}_{n}$ is the $n$th bi-periodic Jacobsthal number and $C_{n}$ is the $n$th bi-periodic Jacobsthal-Lucas number.

Lemma 2.2. Let $\hat{J}_{n}$ and $\hat{C}_{n}$ be the bi-periodic Jacobsthal and the bi-periodic Jacobsthal-Lucas quaternions, respectively. There exist some relations between bi-periodic Jacobsthal and bi-periodic Jacobsthal-Lucas quaternions, for $a, b$ non-zero numbers, as in the followings:

$$
\begin{aligned}
\text { i. } & \hat{J}_{2 n+1}=b \hat{J}_{2 n}+2 \hat{J}_{2 n-1} \text { and } \hat{J}_{2 n}=a \hat{J}_{2 n-1}+2 \hat{J}_{2 n-2}, \\
\text { ii. } & \hat{C}_{2 n+1}=b \hat{C}_{2 n}+2 \hat{C}_{2 n-1} \text { and } \hat{C}_{2 n}=a \hat{C}_{2 n-1}+2 \hat{J}_{2 n-2}, \\
\text { iii. } & \hat{J}_{2 n-1}=(a b+4) \hat{J}_{2 n-3}-4 \hat{J}_{2 n-5} \\
\text { iv. } & \hat{C}_{2 n-1}=(a b+4) \hat{C}_{2 n-3}-4 \hat{C}_{2 n-5} \\
\text { v. } & \hat{C}_{n}=2 \hat{J}_{n-1}+\hat{J}_{n+1} \\
\text { vi. } & (a b+8) \hat{J}_{n}=2 \hat{C}_{n-1}+\hat{C}_{n+1} .
\end{aligned}
$$

Proof. By using definitions of the bi-periodic Jacobsthal and Jacobsthal-Lucas sequences, we obtain the results (i) and (ii).

$$
\text { iii. } \begin{aligned}
\hat{J}_{2 n-1} & =b \hat{J}_{2 n-2}+2 \hat{J}_{2 n-3}=b\left(a \hat{J}_{2 n-3}+2 \hat{J}_{2 n-4}\right)+2 \hat{J}_{2 n-3} \\
& =a b \hat{J}_{2 n-3}+2\left(\hat{J}_{2 n-3}-2 \hat{J}_{2 n-5}\right)+2 \hat{J}_{2 n-3}=(a b+4) \hat{J}_{2 n-3}-4 \hat{J}_{2 n-5} . \\
\text { iv. } \quad \hat{C}_{2 n-1} & =a \hat{C}_{2 n-2}+2 \hat{C}_{2 n-3}=a\left(b \hat{C}_{2 n-3}+2 \hat{C}_{2 n-4}\right)+2 \hat{C}_{2 n-3} \\
& =a b \hat{C}_{2 n-3}+2\left(\hat{C}_{2 n-3}-2 \hat{C}_{2 n-5}\right)+2 \hat{C}_{2 n-3}=(a b+4) \hat{C}_{2 n-3}-4 \hat{C}_{2 n-5} .
\end{aligned}
$$

Other proofs can be obtained using the equations $C_{n}=2 \hat{\jmath}_{n-1}+\hat{\jmath}_{n+1}$ and $(a b+8) \hat{\jmath}_{n}=2 C_{n-1}+C_{n+1}$.

Theorem 2.3. The generating functions for the bi- periodic Jacobsthal and Jacobsthal-Lucas quaternions are

$$
\begin{gathered}
G(x)=\frac{\hat{J}_{0}+\hat{J}_{1} x-b \hat{j}_{0} x}{\left(1-b x-2 x^{2}\right)}+(a-b) x \frac{\hat{J}_{1} x+\hat{J}_{3} x^{3}-(a b+4) \hat{J}_{1} x^{3}}{\left(1-b x-2 x^{2}\right)\left(1-(a b+4) x^{2}+4 x^{4}\right)}, \\
H(x)=\frac{\hat{C}_{0}+\hat{C}_{1} x-b \hat{C}_{0} x}{\left(1-a x-2 x^{2}\right)}-(a-b) x \frac{\hat{C}_{1} x+\hat{C}_{3} x^{3}-(a b+4) \hat{C}_{1} x^{3}}{\left(1-a x-2 x^{2}\right)\left(1-(a b+4) x^{2}+4 x^{4}\right)^{\prime}}
\end{gathered}
$$

respectively. 
Proof. The generating function for $\hat{J}_{n}$ is

$$
G(x)=\sum_{n=0}^{\infty} \hat{J}_{n} x^{n}=\hat{J}_{0}+\hat{J}_{1} x+\hat{J}_{2} x^{2}+\cdots+\hat{J}_{n} x^{n}+\cdots,
$$

By multiplying both sides of this equation by $-b x$ and $-2 x^{2}$, we obtain

$$
\begin{aligned}
& -b x G(x)=-b \sum_{n=0}^{\infty} \hat{J}_{n} x^{n+1}=-b \hat{J}_{0} x-b \hat{\jmath}_{1} x^{2}-b \hat{J}_{2} x^{3}-\cdots-b \hat{J}_{n} x^{n+1}-\cdots \\
& -2 x^{2} G(x)=-2 \sum_{n=0}^{\infty} \hat{J}_{n} x^{n+2}=-2 \hat{J}_{0} x^{2}-2 \hat{J}_{1} x^{3}-2 \hat{J}_{2} x^{4}-\cdots-2 \hat{J}_{n} x^{n+2}-\cdots
\end{aligned}
$$

By using Definition 2.1 and $\hat{J}_{2 n+1}=b \hat{J}_{2 n}+2 \hat{J}_{2 n-1}$ in Lemma 2.2, when adding these equations, we obtain

$$
\begin{aligned}
& \left(1-b x-2 x^{2}\right) G(x)=\hat{J}_{0}+\hat{J}_{1} x-b \hat{J}_{0} x+\left(\hat{J}_{2}-b \hat{J}_{1}-2 \hat{J}_{0}\right) x^{2}+\left(\hat{J}_{4}-b \hat{J}_{3}-2 \hat{J}_{2}\right) x^{4}+\cdots \\
& +\left(\hat{J}_{2 n}-b \hat{J}_{2 n-1}-2 \hat{J}_{2 n-2}\right) x^{2 n}+\cdots
\end{aligned}
$$

From Lemma 2.2, since we know that $\hat{J}_{2 n}=a \hat{J}_{2 n-1}+2 \hat{J}_{2 n-2}$, we have

$$
\left(1-b x-2 x^{2}\right) G(x)=\hat{J}_{0}+\hat{J}_{1} x-b \hat{J}_{0} x+\sum_{n=1}^{\infty}(a-b) \hat{J}_{2 n-1} x^{2 n}
$$

and so

$$
\left(1-b x-2 x^{2}\right) G(x)=\hat{J}_{0}+\hat{J}_{1} x-b \hat{J}_{0} x+(a-b) x \sum_{n=1}^{\infty} \hat{J}_{2 n-1} x^{2 n-1} .
$$

By Lemma 2.2, we have $\hat{J}_{2 n-1}=(a b+4) \hat{J}_{2 n-3}-4 \hat{J}_{2 n-5}$. Hence, by adding the following equations, we obtain $\sum_{n=1}^{\infty} \hat{J}_{2 n-1} x^{2 n-1}$ as follow:

$$
\begin{gathered}
\sum_{n=1}^{\infty} \hat{J}_{2 n-1} x^{2 n-1}=\hat{J}_{1} x+\hat{J}_{3} x^{3}+\cdots+\hat{J}_{2 n-1} x^{n 2 n-1}+\cdots \\
-(a b+4) x^{2} \sum_{n=2}^{\infty} \hat{J}_{2 n-3} x^{2 n-3}=-(a b+4) \hat{J}_{1} x^{3}-(a b+4) \hat{J}_{3} x^{5}-\cdots-(a b+4) \hat{J}_{2 n-3} x^{2 n-1}-\cdots \\
4 x^{4} \sum_{n=3}^{\infty} \hat{J}_{2 n-5} x^{2 n-5}=4 \hat{J}_{1} x^{5}+4 \hat{J}_{3} x^{7}+\cdots+4 \hat{J}_{2 n-5} x^{2 n-1}+\cdots
\end{gathered}
$$

Hence, we get

$$
\sum_{n=1}^{\infty} \hat{J}_{2 n-1} x^{2 n-1}=\frac{\hat{J}_{1} x+\hat{J}_{3} x^{3}-(a b+4) \hat{J}_{1} x^{3}}{\left(1-(a b+4) x^{2}+4 x^{4}\right)} .
$$

By rewriting (2.2) in equation (2.1), we obtain

$$
\begin{gathered}
\left(1-b x-2 x^{2}\right) G(x)=\hat{J}_{0}+\hat{J}_{1} x-b \hat{J}_{0} x+(a-b) x \frac{\hat{J}_{1} x+\hat{J}_{3} x^{3}-(a b+4) \hat{J}_{1} x^{3}}{\left(1-(a b+4) x^{2}+4 x^{4}\right)}, \\
G(x)=\frac{\hat{J}_{0}+\hat{J}_{1} x-b \hat{J}_{0} x}{\left(1-b x-2 x^{2}\right)}+(a-b) x \frac{\hat{J}_{1} x+\hat{J}_{3} x^{3}-(a b+4) \hat{J}_{1} x^{3}}{\left(1-b x-2 x^{2}\right)\left(1-(a b+4) x^{2}+4 x^{4}\right)} .
\end{gathered}
$$

Similarly, we obtain the generating function for the bi- periodic Jacobsthal-Lucas quaternion. 
Theorem 2.4. The Binet formulas for the bi-periodic Jacobsthal and bi-periodic Jacobsthal-Lucas quaternions are, respectively,

$$
\hat{J}_{n}=\left\{\begin{array}{ll}
\frac{1}{(a b)^{\left\lfloor\frac{n}{2}\right\rfloor} \frac{\alpha^{*} \alpha^{n}-\beta^{*} \beta^{n}}{\alpha-\beta}}, & n \text { is even } \\
\frac{1}{(a b)^{\left\lfloor\frac{n}{2}\right\rfloor} \frac{\alpha^{* *} \alpha^{n}-\beta^{* *} \beta^{n}}{\alpha-\beta}} & n \text { is odd }
\end{array} \quad \text { and } \quad \hat{C}_{n}=\left\{\begin{array}{ll}
\frac{1}{(a b)^{\left\lfloor\frac{n+1}{2}\right\rfloor}} \alpha^{* *} \alpha^{n}+\beta^{* *} \beta^{n}, & n \text { is even } \\
\frac{1}{(a b)^{\left\lfloor\frac{n+1}{2}\right\rfloor}} \alpha^{*} \alpha^{n}+\beta^{*} \beta^{n}, & n \text { is odd }
\end{array},\right.\right.
$$

where $\quad \alpha^{*}:=\sum_{i=0}^{3}\left(\frac{a^{\epsilon(i+1)}}{(a b)^{\left.\frac{i}{2}\right\rfloor}}\right) \alpha^{i} e_{i} \quad, \quad \beta^{*}:=\sum_{i=0}^{3}\left(\frac{a^{\epsilon(i+1)}}{(a b)^{\left\lfloor\frac{i}{2}\right\rfloor}}\right) \beta^{i} e_{i} \quad, \quad \alpha^{* *}:=\sum_{i=0}^{3}\left(\frac{a^{\epsilon(i)}}{(a b)^{\left\lfloor\frac{i+1}{2}\right\rfloor}}\right) \alpha^{i} e_{i} \quad$ and $\beta^{* *}:=\sum_{i=0}^{3}\left(\frac{a^{\epsilon(i)}}{(a b)^{\left.\mid \frac{i+1}{2}\right]}}\right) \beta^{i} e_{i}$

Proof. By using Definition 2.1 and the Binet formulas of bi-periodic Jacobsthal and bi-periodic Jacobsthal-Lucas numbers, we obtain the desired results.

Theorem 2.5. (Catalan's identity) For nonnegative integer numbers $n$ and $r$, with $n \geq r$, we have

$$
\begin{gathered}
\hat{J}_{2(n-r)} \hat{J}_{2(n+r)}-\hat{J}_{2 n}^{2}=\frac{2^{2 n-2 r} \hat{J}_{2 r}}{(\alpha-\beta) a(a b)^{r}}\left(\alpha^{*} \beta^{*} \beta^{2 r}-\beta^{*} \alpha^{*} \alpha^{2 r}\right), \\
\hat{C}_{2(n-r)} \hat{C}_{2(n+r)}-\hat{C}_{2 n}^{2}=\frac{2^{2 n-2 r} \hat{J}_{2 r}}{a(a b)^{r}}(\alpha-\beta)\left(\beta^{* *} \alpha^{* *} \alpha^{2 r}-\alpha^{* *} \beta^{* *} \beta^{2 r}\right) .
\end{gathered}
$$

Proof.

$$
\begin{aligned}
& \hat{J}_{2(n-r)} \hat{J}_{2(n+r)}-\hat{J}_{2 n}^{2}=\left(\frac{1}{(a b)^{n-r}} \frac{\alpha^{*} \alpha^{2 n-2 r}-\beta^{*} \beta^{2 n-2 r}}{\alpha-\beta}\right)\left(\frac{1}{(a b)^{n+r}} \frac{\alpha^{*} \alpha^{2 n+2 r}-\beta^{*} \beta^{2 n+2 r}}{\alpha-\beta}\right)-\left(\frac{1}{(a b)^{n}} \frac{\alpha^{*} \alpha^{2 n}-\beta^{*} \beta^{2 n}}{\alpha-\beta}\right)^{2} \\
& =\frac{1}{(a b)^{2 n}(\alpha-\beta)^{2}}\left(\alpha^{*} \beta^{*}\left((\alpha \beta)^{2 n}-\alpha^{2 n-2 r} \beta^{2 n+2 r}\right)+\beta^{*} \alpha^{*}\left((\alpha \beta)^{2 n}-\alpha^{2 n+2 r} \beta^{2 n-2 r}\right)\right) \\
& =\frac{1}{(a b)^{2 n}(\alpha-\beta)^{2}}(\alpha \beta)^{2 n}\left(\alpha^{*} \beta^{*}\left(1-\frac{\beta^{2 r}}{\alpha^{2 r}}\right)+\beta^{*} \alpha^{*}\left(1-\frac{\alpha^{2 r}}{\beta^{2 r}}\right)\right) \\
& =\frac{2^{2 n}}{(\alpha-\beta)^{2}}\left(\alpha^{*} \beta^{*} \frac{\alpha^{2 r}-\beta^{2 r}}{\alpha^{2 r}}\right)-\frac{2^{2 n}}{(\alpha-\beta)^{2}}\left(\beta^{*} \alpha^{*} \frac{\alpha^{2 r}-\beta^{2 r}}{\beta^{2 r}}\right) \\
& =\frac{2^{2 n}(a b)^{r} \hat{\jmath}_{2 r}}{a(\alpha-\beta)}\left(\frac{\alpha^{*} \beta^{*}}{\alpha^{2 r}}-\frac{\beta^{*} \alpha^{*}}{\beta^{2 r}}\right)=\frac{2^{2 n-2 r} \hat{\jmath}_{2 r}}{(\alpha-\beta) a(a b)^{r}}\left(\alpha^{*} \beta^{*} \beta^{2 r}-\beta^{*} \alpha^{*} \alpha^{2 r}\right) .
\end{aligned}
$$

Similarly, we prove that

$$
\hat{C}_{2(n-r)} \hat{C}_{2(n+r)}-\hat{C}_{2 n}^{2}=\frac{2^{2 n-2 r} \hat{J}_{2 r}}{a(a b)^{r}}(\alpha-\beta)\left(\beta^{* *} \alpha^{* *} \alpha^{2 r}-\alpha^{* *} \beta^{* *} \beta^{2 r}\right) .
$$

Theorem 2.6. (Cassini's identity) For $n \geq 1$, we have

$$
\begin{gathered}
\hat{J}_{2(n-1)} \hat{J}_{2(n+1)}-\hat{J}_{2 n}^{2}=\frac{2^{2 n-2}}{a b(\alpha-\beta)}\left(\alpha^{*} \beta^{*} \beta^{2}-\beta^{*} \alpha^{*} \alpha^{2}\right), \\
\hat{C}_{2(n-1)} \hat{C}_{2(n+1)}-\hat{C}_{2 n}^{2}=\frac{2^{2 n-2}}{a b}(\alpha-\beta)\left(\beta^{* *} \alpha^{* *} \alpha^{2}-\alpha^{* *} \beta^{* *} \beta^{2}\right) .
\end{gathered}
$$

Since Cassini's identity is a special case of Catalan's identity, the proof is seen by taking $r=1$.

Theorem 2.7. (D'ocagne's property) For nonnegative integer numbers $n$ and $m$, with $m \geq n$, we have

$$
\begin{gathered}
\hat{J}_{2 m} \hat{J}_{2(n+1)}-\hat{J}_{2(m+1)} \hat{J}_{2 n}=\frac{2^{2 n}(a b)^{n-m}\left(\alpha^{*} \beta^{*} \alpha^{2 m-2 n}-\beta^{*} \alpha^{*} \beta^{2 m-2 n}\right)}{(\alpha-\beta)}, \\
\hat{C}_{2 m} \hat{C}_{2(n+1)}-\hat{C}_{2(m+1)} \hat{C}_{2 n}=2^{2 n}(a b)^{n-m}(\alpha-\beta)\left(\beta^{* *} \alpha^{* *} \beta^{2 m-2 n}-\alpha^{* *} \beta^{* *} \alpha^{2 m-2 n}\right) .
\end{gathered}
$$


Proof.

$$
\begin{aligned}
& \hat{J}_{2 m} \hat{J}_{2(n+1)}-\hat{J}_{2(m+1)} \hat{J}_{2 n}=\frac{1}{(a b)^{m}}\left(\frac{\alpha^{*} \alpha^{2 m}-\beta^{*} \beta^{2 m}}{\alpha-\beta}\right) \frac{1}{(a b)^{n+1}}\left(\frac{\alpha^{*} \alpha^{2 n+2}-\beta^{*} \beta^{2 n+2}}{\alpha-\beta}\right) \\
& -\frac{1}{(a b)^{m+1}}\left(\frac{\alpha^{*} \alpha^{2 m+2}-\beta^{*} \beta^{2 m+2}}{\alpha-\beta}\right) \frac{1}{(a b)^{n}}\left(\frac{\alpha^{*} \alpha^{2 n}-\beta^{*} \beta^{2 n}}{\alpha-\beta}\right) \\
& =\frac{1}{(a b)^{m+n+1}(\alpha-\beta)^{2}}\left(\alpha^{*} \beta^{*}\left(\alpha^{2 m+2} \beta^{2 n}-\alpha^{2 m} \beta^{2 n+2}\right)+\beta^{*} \alpha^{*}\left(\alpha^{2 n} \beta^{2 m+2}-\alpha^{2 n+2} \beta^{2 m}\right)\right) \\
& =\frac{2^{2 n}(a b)^{n-m-1}}{(\alpha-\beta)^{2}}\left(\alpha^{*} \beta^{*}\left(\alpha^{2 m-2 n+2}-\alpha^{2 m-2 n} \beta^{2}\right)+\beta^{*} \alpha^{*}\left(\beta^{2 m-2 n+2}-\alpha^{2} \beta^{2 m-2 n}\right)\right) \\
& =\frac{2^{2 n}(a b)^{n-m-1}}{(\alpha-\beta)^{2}}\left(\alpha^{*} \beta^{*} \alpha^{2 m-2 n}\left(\alpha^{2}-\beta^{2}\right)+\beta^{*} \alpha^{*} \beta^{2 m-2 n}\left(\beta^{2}-\alpha^{2}\right)\right) \\
& =\frac{2^{2 n}(a b)^{n-m}\left(\alpha^{*} \beta^{*} \alpha^{2 m-2 n}-\beta^{*} \alpha^{*} \beta^{2 m-2 n}\right)}{(\alpha-\beta)} .
\end{aligned}
$$

D'ocagne's property for the bi-periodic Jacobsthal-Lucas quaternion can be proven similarly.

Theorem 2.8. For $n \geq 1$ and nonnegative even integer $r$ such that $r \leq n$, we have

$$
\begin{gathered}
\hat{J}_{n+r} \hat{J}_{n-r}-\hat{J}_{n}^{2}=\left\{\begin{array}{ll}
-\frac{2^{n-r} \hat{\jmath}_{r}}{a(a b)^{r / 2}(\alpha-\beta)}\left(\alpha^{*} \beta^{*} \alpha^{r}-\beta^{*} \alpha^{*} \beta^{r}\right), & n \text { is even } \\
\frac{2^{n-r} \hat{\jmath}_{r}}{a(a b)^{(r-2) / 2}(\alpha-\beta)}\left(\alpha^{* *} \beta^{* *} \alpha^{r}-\beta^{* *} \alpha^{* *} \beta^{r}\right), & n \text { is odd }
\end{array},\right. \\
\hat{C}_{n+r} \hat{C}_{n-r}-\hat{C}_{n}^{2}= \begin{cases}\frac{2^{n-r}(\alpha-\beta) \hat{\jmath}_{r}}{a(a b)^{r / 2}}\left(\alpha^{* *} \beta^{* *} \alpha^{r}-\beta^{* *} \alpha^{* *} \beta^{r}\right), & n \text { is even } \\
-\frac{2^{n-r}(\alpha-\beta) \hat{\jmath}_{r}}{a(a b)^{(r+2) / 2}}\left(\alpha^{*} \beta^{*} \alpha^{r}-\beta^{*} \alpha^{*} \beta^{r}\right), & n \text { is odd }\end{cases}
\end{gathered}
$$

Proof. If $n$ is even then we have

$$
\begin{aligned}
\hat{J}_{n+r} \hat{J}_{n-r}-\hat{J}_{n}^{2} & =\frac{1}{(a b)^{n}}\left(\frac{\alpha^{*} \alpha^{n+r}-\beta^{*} \beta^{n+r}}{\alpha-\beta}\right)\left(\frac{\alpha^{*} \alpha^{n-r}-\beta^{*} \beta^{n-r}}{\alpha-\beta}\right)-\frac{1}{(a b)^{n}}\left(\frac{\alpha^{*} \alpha^{n}-\beta^{*} \beta^{n}}{\alpha-\beta}\right)\left(\frac{\alpha^{*} \alpha^{n}-\beta^{*} \beta^{n}}{\alpha-\beta}\right) \\
& =-\frac{2^{n}}{(\alpha-\beta)^{2}}\left(\alpha^{*} \beta^{*}\left(\frac{\alpha^{r}-\beta^{r}}{\beta^{r}}\right)-\beta^{*} \alpha^{*}\left(\frac{\alpha^{r}-\beta^{r}}{\alpha^{r}}\right)\right) \\
& =-\frac{2^{n-r} \hat{\jmath}_{r}}{a(a b)^{r / 2}(\alpha-\beta)}\left(\alpha^{*} \beta^{*} \alpha^{r}-\beta^{*} \alpha^{*} \beta^{r}\right) .
\end{aligned}
$$

If $n$ is odd then we have

$$
\begin{aligned}
& \hat{J}_{n+r} \hat{J}_{n-r}-\hat{J}_{n}^{2}=\frac{1}{(a b)^{\frac{n+r-1}{2}}}\left(\frac{\alpha^{* *} \alpha^{n+r}-\beta^{* *} \beta^{n+r}}{\alpha-\beta}\right) \frac{1}{(a b)^{\frac{n-r-1}{2}}}\left(\frac{\alpha^{* *} \alpha^{n-r}-\beta^{* *} \beta^{n-r}}{\alpha-\beta}\right)-\frac{1}{(a b)^{n-1}}\left(\frac{\alpha^{* *} \alpha^{n}-\beta^{* *} \beta^{n}}{\alpha-\beta}\right)\left(\frac{\alpha^{* *} \alpha^{n}-\beta^{* *} \beta^{n}}{\alpha-\beta}\right) \\
& =\frac{(-2 a b)^{n}}{(a b)^{n-1}(\alpha-\beta)^{2}}\left(\alpha^{* *} \beta^{* *}-\left(\frac{\alpha^{r}-\beta^{r}}{\beta^{r}}\right)+\beta^{* *} \alpha^{* *}\left(\frac{\alpha^{r}-\beta^{r}}{\alpha^{r}}\right)\right)=\frac{2^{n-r} \hat{J}_{r}}{a(a b)^{(r-2) / 2}(\alpha-\beta)}\left(\alpha^{* *} \beta^{* *} \alpha^{r}-\beta^{* *} \alpha^{* *} \beta^{r}\right) .
\end{aligned}
$$

The other part of the proof is calculated using the same method.

Theorem 2.9. For $k$ is even and $l>k$, we have

$$
\begin{aligned}
& \sum_{r=0}^{n}(a b)^{\frac{k r}{2}} \hat{\jmath}_{k r+l}=\frac{\hat{\jmath}_{l}-(a b)^{\frac{k(n+1)}{2}} \hat{\jmath}_{k n+k+l}+2^{k}(a b)^{\frac{k(n+2)}{2}} \hat{\jmath}_{k n+l}-2^{k}(a b)^{\frac{k}{2}} \hat{J}_{l-k}}{1+(2 a b)^{k}-\left(\alpha^{k}+\beta^{k}\right)}, \\
& \sum_{r=0}^{n}(a b)^{\frac{k r}{2}} \hat{C}_{k r+l}=\frac{\hat{C}_{l}-(a b)^{\frac{k(n+1)}{2}} \hat{C}_{k n+k+l}+2^{k}(a b)^{\frac{k(n+2)}{2}} \hat{C}_{k n+l}-2^{k}(a b)^{\frac{k}{2}} \hat{C}_{l-k}}{1+(2 a b)^{k}-\left(\alpha^{k}+\beta^{k}\right)} .
\end{aligned}
$$


Proof. Let $l$ be even. Additionally, we know that $k$ is even with $l>k$. Thus, we obtain as follows:

$$
\begin{aligned}
& \sum_{r=0}^{n}(a b)^{\frac{k r}{2}} \hat{J}_{k r+l}=\sum_{r=0}^{n} \frac{(a b)^{\frac{k r}{2}}}{(a b)^{\frac{k r+l}{2}}} \frac{\alpha^{*} \alpha^{k r+l}-\beta^{*} \beta^{k r+l}}{\alpha-\beta} \\
= & \frac{\alpha^{*} \alpha^{l}}{(a b)^{\frac{l}{2}}(\alpha-\beta)} \sum_{r=0}^{n} \alpha^{k r}-\frac{\beta^{*} \beta^{l}}{(a b)^{\frac{l}{2}}(\alpha-\beta)} \sum_{r=0}^{n} \beta^{k r} \\
= & \frac{\alpha^{*} \alpha^{l}}{(a b)^{\frac{l}{2}}(\alpha-\beta)} \frac{1-(\alpha)^{k n+k}}{1-\alpha^{k}}-\frac{\beta^{*} \beta^{l}}{(a b)^{\frac{l}{2}}(\alpha-\beta)} \frac{1-(\beta)^{k n+k}}{1-\beta^{k}} \\
= & \frac{1}{(a b)^{\frac{l}{2}}(\alpha-\beta)}\left(\frac{\alpha^{*} \alpha^{l}-\alpha^{*} \alpha^{l} \beta^{k}-\alpha^{*} \alpha^{k n+k+l}+\alpha^{*} \alpha^{k n+k+l} \beta^{k}-\beta^{*} \beta^{l}+\beta^{*} \beta^{l} \alpha^{k}+\beta^{*} \beta^{k n+k+l}-\beta^{*} \beta^{k n+k+l} \alpha^{k}}{\left(1-\alpha^{k}\right)\left(1-\beta^{k}\right)}\right) \\
= & \frac{\hat{J}_{l}-(a b)^{\frac{k(n+1)}{2}} \hat{J}_{k n+k+l}+2^{k}(a b)^{\frac{k(n+2)}{2}} \hat{J}_{k n+l}-2^{k}(a b)^{\frac{k}{2}} \hat{J}_{l-k}}{1+(2 a b)^{k}-\left(\alpha^{k}+\beta^{k}\right)} .
\end{aligned}
$$

The equation is provided in the case $l$ is odd. Similarly, we see the other part of the proof for bi-periodic Jacobsthal-Lucas quaternions.

Theorem 2.10. For $k$ is even and $s>k$, we have

$$
\begin{aligned}
& \sum_{r=0}^{\infty}(a b)^{-\left\lfloor\frac{3 k r}{2}\right\rfloor} \hat{J}_{k r+s}=\frac{(a b)^{k}}{(a b)^{3 k}-(a b)^{k}\left(\alpha^{k}+\beta^{k}\right)+2^{k}}\left((a b)^{2 k} \hat{J}_{s}-2^{k}(a b)^{k / 2} \hat{J}_{s-k}\right) \\
& \sum_{r=0}^{\infty}(a b)^{-\left\lfloor\frac{3 k r}{2}\right\rfloor} \hat{C}_{k r+s}=\frac{(a b)^{k}}{(a b)^{3 k}-(a b)^{k}\left(\alpha^{k}+\beta^{k}\right)+2^{k}}\left((a b)^{2 k} \hat{C}_{s}-2^{k}(a b)^{k / 2} \hat{C}_{s-k}\right)
\end{aligned}
$$

Proof. Let $s$ be even, then we obtain

$$
\begin{aligned}
\sum_{r=0}^{\infty}(a b)^{-\left\lfloor\frac{3 k r}{2}\right\rfloor} \hat{J}_{k r+s} & =\sum_{r=0}^{\infty}(a b)^{-\left\lfloor\frac{3 k r}{2}\right\rfloor} \frac{1}{(a b)^{\left.\frac{k r+s}{2}\right\rfloor} \frac{\alpha^{*} \alpha^{k r+s}-\beta^{*} \beta^{k r+s}}{\alpha-\beta}} \\
& =\frac{\alpha^{*} \alpha^{s}}{(\alpha-\beta)(a b)^{\frac{s}{2}}} \sum_{r=0}^{\infty} \frac{\alpha^{k r}}{(a b)^{2 k r}}-\frac{\beta^{*} \beta^{s}}{(\alpha-\beta)(a b)^{\frac{s}{2}}} \sum_{r=0}^{\infty} \frac{\beta^{k r}}{(a b)^{2 k r}} \\
& =\frac{1}{(\alpha-\beta)(a b)^{\frac{s}{2}}}\left(\alpha^{*} \alpha^{s}\left(\frac{1-\frac{\alpha^{k}}{(a b)^{2 k}}}{1}\right)-\beta^{*} \beta^{s}\left(\frac{1}{1-\frac{\beta^{k}}{(a b)^{2 k}}}\right)\right) \\
& =\frac{(a b)^{2 k}}{(\alpha-\beta)(a b)^{\frac{s}{2}}}\left(\left(\frac{\alpha^{*} \alpha^{s}}{\left.\left.(a b)^{2 k}-\alpha^{k}\right)-\left(\frac{\beta^{*} \beta^{s}}{(a b)^{2 k}-\beta^{k}}\right)\right)}\right)\right. \\
& =\frac{(a b)^{2 k}}{(\alpha-\beta)(a b)^{\frac{s}{2}}}\left(\frac{(a b)^{2 k} \alpha^{*} \alpha^{s}-\alpha^{*} \alpha^{s} \beta^{k}-(a b)^{2 k} \beta^{*} \beta^{s}+\beta^{*} \alpha^{k} \beta^{s}}{(a b)^{k}\left((a b)^{3 k}-(a b)^{k}\left(\alpha^{k}+\beta^{k}\right)+2^{k}\right)}\right)
\end{aligned}
$$




$$
\begin{aligned}
& =\frac{(a b)^{k}}{(\alpha-\beta)(a b)^{\frac{s}{2}}}\left(\frac{(a b)^{2 k}\left(\alpha^{*} \alpha^{s}-\beta^{*} \beta^{s}\right)-(\alpha \beta)^{k}\left(\alpha^{*} \alpha^{s-k}-\beta^{*} \beta^{s-k}\right)}{(a b)^{3 k}-(a b)^{k}\left(\alpha^{k}+\beta^{k}\right)+2^{k}}\right) \\
& =\frac{(a b)^{k}}{(a b)^{3 k}-(a b)^{k}\left(\alpha^{k}+\beta^{k}\right)+2^{k}}\left(\frac{(a b)^{2 k}\left(\alpha^{*} \alpha^{s}-\beta^{*} \beta^{s}\right)-2^{k}(a b)^{k}\left(\alpha^{*} \alpha^{s-k}-\beta^{*} \beta^{s-k}\right)}{(\alpha-\beta)(a b)^{\frac{s}{2}}}\right) \\
& =\frac{(a b)^{k}}{(a b)^{3 k}-(a b)^{k}\left(\alpha^{k}+\beta^{k}\right)+2^{k}}\left((a b)^{2 k} \hat{J}_{s}-2^{k}(a b)^{k / 2} \hat{J}_{s-k}\right) .
\end{aligned}
$$

When $s$ is odd, the proof is calculated using the same method.

Similarly, we see the other part of the proof by using the Binet formula for bi-periodic Jacobsthal-Lucas quaternion.

Theorem 2.11. For $n \geq r \geq 0$ such that $n$ is even, we have the following summation formulas:

$$
\begin{aligned}
& \sum_{r=0}^{n}\left(\begin{array}{l}
n \\
r
\end{array}\right)(-1)^{r} 2^{n-r}(a b)^{-n / 2} \hat{J}_{2 r}=\hat{J}_{n}, \\
& \sum_{r=0}^{n}\left(\begin{array}{l}
n \\
r
\end{array}\right)(-1)^{r} 2^{n-r}(a b)^{-n / 2} \hat{C}_{2 r}=\hat{C}_{n} .
\end{aligned}
$$

Proof.

$$
\begin{aligned}
\sum_{r=0}^{n}\left(\begin{array}{l}
n \\
r
\end{array}\right)(-1)^{r} 2^{n-r}(a b)^{-n / 2} \hat{J}_{2 r} & =\sum_{r=0}^{n}\left(\begin{array}{l}
n \\
r
\end{array}\right)(-1)^{r} 2^{n-r}(a b)^{-n / 2} \frac{1}{(a b)^{r}} \frac{\alpha^{*} \alpha^{2 r}-\beta^{*} \beta^{2 r}}{\alpha-\beta} \\
& =\frac{(a b)^{-n / 2}}{\alpha-\beta}\left[\alpha^{*}\left(\sum_{r=0}^{n}\left(\begin{array}{l}
n \\
r
\end{array}\right)(-1)^{r} 2^{n-r}\left(\frac{\alpha^{2}}{a b}\right)^{r}\right)-\beta^{*}\left(\sum_{r=0}^{n}\left(\begin{array}{l}
n \\
r
\end{array}\right)(-1)^{r} 2^{n-r}\left(\frac{\beta^{2}}{a b}\right)^{r}\right)\right] \\
& =\frac{(a b)^{-n / 2}}{\alpha-\beta}\left(\alpha^{*}\left(\frac{\alpha^{2}}{a b}-2\right)^{n}-\beta^{*}\left(\frac{\beta^{2}}{a b}-2\right)^{n}\right) \\
& =\frac{(a b)^{-n / 2}}{\alpha-\beta}\left(\alpha^{*} \alpha^{n}-\beta^{*} \beta^{n}\right) \\
& =\frac{1}{(a b)^{n / 2}}\left(\frac{\alpha^{*} \alpha^{n}-\beta^{*} \beta^{n}}{\alpha-\beta}\right)=\hat{J}_{n} .
\end{aligned}
$$

For the bi-periodic Jacobsthal-Lucas quaternion, desired result can be seen.

Finally, we give the relationships between bi-periodic Jacobsthal quaternion and bi-periodic Jacobsthal-Lucas quaternion. In Theorem 2.12, the identities given in Lemma 2.2 ( $\mathrm{v}$ and vi) will be proven by using the Binet Formula.

Theorem 2.12. There are the relationships between bi-periodic Jacobsthal quaternion $\hat{J}_{n}$ and bi-periodic Jacobsthal-Lucas quaternion $\hat{C}_{n}$ as follows:

$$
\begin{gathered}
\hat{J}_{n+1}+2 \hat{J}_{n-1}=\hat{C}_{n}, \\
\hat{C}_{n+1}+2 \hat{C}_{n-1}=(a b+8) \hat{J}_{n} .
\end{gathered}
$$

Proof. For $n$ is even, by using the Binet formulas, we obtain

$$
\begin{aligned}
\hat{J}_{n+1}+2 \hat{\jmath}_{n-1} & =\frac{1}{(a b)^{n / 2}}\left(\frac{\alpha^{* *} \alpha^{n+1}-\beta^{* *} \beta^{n+1}}{\alpha-\beta}\right)+\frac{2}{(a b)^{(n-2) / 2}}\left(\frac{\alpha^{* *} \alpha^{n-1}-\beta^{* *} \beta^{n-1}}{\alpha-\beta}\right) \\
& =\frac{1}{(a b)^{n / 2}}\left(\frac{\alpha^{* *} \alpha^{n+1}-\beta^{* *} \beta^{n+1}}{\alpha-\beta}-\alpha \beta \frac{\alpha^{* *} \alpha^{n-1}-\beta^{* *} \beta^{n-1}}{\alpha-\beta}\right) \\
& =\frac{1}{(a b)^{n / 2}}\left(\frac{\alpha^{* *} \alpha^{n}(\alpha-\beta)-\beta^{* *} \beta^{n}(\beta-\alpha)}{\alpha-\beta}\right)
\end{aligned}
$$


For $n$ is odd,

$$
=\frac{1}{(a b)^{\left\lfloor\frac{n}{2}\right\rfloor}}\left(\alpha^{* *} \alpha^{n}+\beta^{* *} \beta^{n}\right)=\hat{C}_{n} .
$$

$$
\begin{aligned}
\hat{J}_{n+1}+2 \hat{J}_{n-1} & =\frac{1}{(a b)^{(n+1) / 2}}\left(\frac{\alpha^{*} \alpha^{n+1}-\beta^{*} \beta^{n+1}}{\alpha-\beta}\right)+\frac{2}{(a b)^{(n-1) / 2}}\left(\frac{\alpha^{*} \alpha^{n-1}-\beta^{*} \beta^{n-1}}{\alpha-\beta}\right) \\
& =\frac{1}{(a b)^{(n+1) / 2}}\left(\frac{\alpha^{*} \alpha^{n+1}-\beta^{*} \beta^{n+1}}{\alpha-\beta}-\alpha \beta \frac{\alpha^{*} \alpha^{n-1}-\beta^{*} \beta^{n-1}}{\alpha-\beta}\right) \\
& =\frac{1}{(a b)^{(n+1) / 2}}\left(\frac{\alpha^{*} \alpha^{n}(\alpha-\beta)-\beta^{*} \beta^{n}(\beta-\alpha)}{\alpha-\beta}\right) \\
& =\frac{1}{(a b)^{\left\lfloor\frac{n+1}{2}\right\rfloor}}\left(\alpha^{*} \alpha^{n}+\beta^{*} \beta^{n}\right)=\hat{C}_{n} .
\end{aligned}
$$

Similarly, if $n$ is even

$$
\begin{aligned}
\hat{C}_{n+1}+2 \hat{C}_{n-1} & =\frac{1}{(a b)^{(n+2) / 2}}\left(\alpha^{*} \alpha^{n+1}+\beta^{*} \beta^{n+1}\right)+\frac{2}{(a b)^{n / 2}}\left(\alpha^{*} \alpha^{n-1}+\beta^{*} \beta^{n-1}\right) \\
& =\frac{1}{(a b)^{(n+2) / 2}}\left(\alpha^{*} \alpha^{n+1}+\beta^{*} \beta^{n+1}-\alpha \beta\left(\alpha^{*} \alpha^{n-1}+\beta^{*} \beta^{n-1}\right)\right) \\
& =\frac{1}{(a b)^{(n+2) / 2}}\left(\alpha^{*} \alpha^{n}(\alpha-\beta)+\beta^{*} \beta^{n}(\beta-\alpha)\right) \\
& =\frac{\alpha-\beta}{(a b)^{(n+2) / 2}}\left(\alpha^{*} \alpha^{n}-\beta^{*} \beta^{n}\right) \\
& =\frac{(\alpha-\beta)^{2}}{a b} \hat{J}_{n}=(a b+8) \hat{J}_{n} .
\end{aligned}
$$

If $n$ is odd, we have $\hat{C}_{n+1}+2 \hat{C}_{n-1}=(a b+8) \hat{J}_{n}$.

Theorem 2.13. For $n$ is odd, we have

$$
\hat{J}_{n}^{2}=(a b+8)\left(\hat{C}_{2 n}+2^{n}\left(\alpha^{* *} \beta^{* *}+\beta^{* *} \alpha^{* *}\right)\right) .
$$

Proof. If $n$ is odd, then we have

$$
\begin{aligned}
\hat{J}_{n}^{2} & =\frac{1}{(a b)^{n-1}}\left(\frac{\alpha^{* *} \alpha^{n}-\beta^{* *} \beta^{n}}{\alpha-\beta}\right)^{2} \\
& =\frac{1}{(a b)^{n-1}(\alpha-\beta)^{2}}\left(\alpha^{* *} \alpha^{2 n}-\alpha^{* *} \beta^{* *}(\alpha \beta)^{n}-\beta^{* *} \alpha^{* *}(\alpha \beta)^{n}+\beta^{* *} \beta^{2 n}\right) \\
& =\frac{a b}{(\alpha-\beta)^{2}}\left(\hat{C}_{2 n}+2^{n}\left(\alpha^{* *} \beta^{* *}+\beta^{* *} \alpha^{* *}\right)\right) \\
& =(a b+8)\left(\hat{C}_{2 n}+2^{n}\left(\alpha^{* *} \beta^{* *}+\beta^{* *} \alpha^{* *}\right)\right)
\end{aligned}
$$

\section{References}

Bilgici, G. (2014). Two generalizations of Lucas sequence. Appl. Math. Comput., 245 , 526-538. https://doi.org/10.1016/j.amc.2014.07.111

Edson, M., \& Yayenie, O. (2009). A new generalization of Fibonacci sequences and the extended Binet's formula. Integer Electron. J. Comb. Number Theory, 9, 639-654. https://doi.org/10.1515/INTEG.2009.051

El Naschie, M. S. (2005). Deriving the essential features of the standard model from the general theory of relativity. Chaos, Solitons \& Fractals, 24(4), 941-946. http://dx.doi.org/10.1016/j.chaos.2004.10.001 
El Naschie, M. S. (2007). The Fibonacci Code behind Super Strings and P-Branes: An Answer to M. Kaku's Fundamental Question. Chaos, Solitons \& Fractals, 31, 537-547. http://dx.doi.org/10.1016/j.chaos.2006.07.001

Gül, K. (2018). On the $k$-Pell quaternions and the $k$-Pell-Lucas quaternions. Iğdır Univ. J. Inst. Sci. \& Tech., 8(1), 23-35. https://dx.doi.org/10.21597/jist.407804

Halıcı, S. (2012). On Fibonacci quaternions. Advences in Applied Clifford Algebras, 22, $321-327$. https://doi.org/10.1007/s00006-011-0317-1

Hamilton, W. R. (1866). Elements of quaternion. London: Longmans, Green \& Company. https://doi.org/10.1017/CBO9780511707162

Horadam, A. F. (1961). A generalized Fibonacci sequence. Math. Mag., 68, 455-459. https://doi.org/ 10.2307/2311099

Horadam, A. F. (1971). Pell identities. Fibonacci Quart., 9, 245-252.

Horadam, A. F. (1993). Quaternion recurrence relations. Ulam Quart., 2, 23-33.

Horadam, A. F. (1996). Jacobsthal representation numbers. Fibonacci Quart., 34, 40-54.

Kilic, E., \& Stakhov, A. P. (2009). On the Fibonacci and Lucas p-numbers, their sums, families of bipartite graphs and permanents of certain matrices. Chaos, Solitons \& Fractals, 40, $2210-\quad 2221$. https://doi.org/10.1016/j.chaos.2007.10.007

Koshy, T. (2001). Fibonacci and Lucas Numbers with Applications, Newyork; A Wiley-Interscience Publication. https://doi.org/10.1002/9781118033067

Stakhov, A. P. (2005). The Generalized Principle of the Golden Section and Its Applications in Mathematics, Science and Engineering. Chaos, Solitons \& Fractals, 26, 263-289. http://dx.doi.org/10.1016/j.chaos.2005.01.038

Szynal-Liana, A., \& Wloch, I. (2016). A note on Jacobsthal quaternions. Adv. Appl. Clifford Algebras, 26(1), $441-447$. https://doi.org/ 10.1007/s00006-015-0622-1

Tan, E., Y1lmaz, S., \& Sahin, M. (2016a). A note on bi-periodic Fibonacci and Lucas quaternions. Chaos Solitons \& Fractals, 85, 138-142. https://doi.org/ 10.1016/j.chaos.2016.01.025

Tan, E., Y1lmaz, S., \& Sahin, M. (2016b). On a new generalization of Fibonacci quaternions. Chaos Solitons \& Fractals, 82, 1-4. https://doi.org/10.1016/j.chaos.2015.10.021

Tasc1, D. (2017). On $k$-Jacobsthal and $k$-Jacobsthal-Lucas quaternions. Journal of Science and Arts, 3, $469-476$.

Uygun, Ş. (2018). A New Generalization of Jacobsthal Lucas Numbers. Asian Journal of Mathematics and Physics, 2(1), 14-21.

Uygun, Ş., \& Owusu, E. (2016). A New Generalization of Jacobsthal Numbers. Journal of Mathematical Analysis, 7(4), 28-39.

Uygun, Ş., \& Owusu, E. (2017). Matrix representation of bi-periodic Jacobsthal sequence. https://arxiv.org/abs/1702.00604.

Yayenie, O. (2011). A note on generalized Fibonacci sequence. Appl. Math. Comput., 217, 5603-5611. https://doi.org/10.1016/j.amc.2010.12.038

\section{Copyrights}

Copyright for this article is retained by the author(s), with first publication rights granted to the journal.

This is an open-access article distributed under the terms and conditions of the Creative Commons Attribution license (http://creativecommons.org/licenses/by/4.0/). 